\title{
Small Dense Low-Density Lipoprotein: Biomarker or Potential Drug Target?
}

\author{
Basabdatta Samanta ${ }^{1}$ \\ ${ }^{1}$ Department of Biochemistry, Burdwan Medical College, West \\ Bengal, India
}

\begin{abstract}
Address for correspondence Basabdatta Samanta, MBBS, MD, DNB, Department of Biochemistry, Burdwan Medical College, Burdwan 713104, West Bengal, India (e-mail: basab_s@yahoo.com).
\end{abstract}

\begin{abstract}
Ischemic heart disease is currently an epidemic affecting individuals worldwide. Increased incidence along with earlier onset of disease has led to the constant search for biomarkers that will help in earlier identification and treatment of at risk individuals. Small dense low-density lipoprotein (sdLDL) is the atherogenic subtype of low-density lipoprotein (LDL). It is smaller in size and higher in density in comparison to other LDL subtypes. Higher levels of sdLDL have been found to be associated with increased incidence of ischemic heart disease and adverse outcomes. Properties including decreased resistance to oxidative stress and prolonged residence time in the circulation account for its increased atherogenic potential. Hence intervention approaches targeting sdLDL directly in at risk individuals may be beneficial.

Genetic, lifestyle, and environmental factors affect sdLDL levels. But the main determining factor is the level of triglycerides (TGs). Higher TG levels are associated with higher levels of very low density lipoprotein (VLDL) 1 and sdLDL. Various drugs

\section{Keywords}

- ischemic heart disease

- small dense lowdensity lipoprotein

- atherogenicity

- drug target have been used for targeting sdLDL with varying outcomes; drugs tried out include statins, fibrates, niacin, cholesterol ester transfer protein inhibitors and sodium-glucose co-transporter- 2 inhibitors. Future prospects include modification of enzymes involved in fatty acid and TG synthesis, for example, lipoprotein lipase and acyl CoA carboxylase. However, further research is still necessary to draw clear guidelines for sdLDL reduction therapy in coronary artery disease treatment and prevention.
\end{abstract}

\section{Introduction}

Cardiovascular disease (CVD), including coronary heart disease (CHD) and coronary artery disease (CAD), is a major cause of morbidity and mortality worldwide. According to the 2016 Heart Disease and Stroke Statistics update of the American Heart Association, in the United States, 15.5 million people $\geq 20$ years of age have CHD. ${ }^{1}$ The prevalence increases with age for both men and women. In India the prevalence of CHD has increased fourfold in the past 40 years. ${ }^{2}$ The Registrar General of India reported that CHD-related adult deaths have increased from 26\% in 2001 to 2003 to $32 \%$ in 2010 to 2013. ${ }^{3}$ Gupta et al reported that cardiovascular risk factors among Indians increase exponentially in the age group of 30 to 39 years. ${ }^{4}$ Covering $24.8 \%$, CVD is the leading cause of death throughout India, including both rural and urban areas. ${ }^{5}$

Increasing incidence combined with earlier onset has led to the constant search for biomarkers which will help in identifying at risk individuals at an early stage. Small dense LDL (sdLDL) is the smaller and denser subtype of LDL. Higher levels of sdLDL have been found to be associated with increased incidence of cardiovascular events. ${ }^{6}$ Hence sdLDL estimation could be used as a means of identification of high-risk individuals. Early intervention using drug therapy for reduction of sdLDL levels could ultimately lead to a better outcome for these people. 


\section{Cardiovascular Risk Assessment}

Risk factors which increase the probability of CHD include diabetes, hypertension, central obesity, physical inactivity, smoking, psychosocial factors, dyslipidemias, and genetic predisposition. The INTERHEART study reported that more than $90 \%$ of the risk factors for CHD were measurable and modifiable. ${ }^{7}$ Among the lipoproteins, traditionally high-density lipoprotein (HDL) is considered as "good cholesterol" and LDL as "bad cholesterol." Longitudinal studies had established the role of LDL as a risk factor for CHD occurrence, recurrence, and fatal outcome. Clinical trials proved that control of elevated LDL in high-risk patients was a very cost effective and efficient means of cardiovascular risk reduction. ${ }^{8}$ Hence lipid profile, in particular LDL, is routinely used by clinicians as a tool for cardiovascular risk assessment.

Other studies, however, found that LDL is not elevated in all those patients suffering from CAD. ${ }^{9}$ Moreover, though lipid lowering agents are widely used in clinical practice as a treatment component in $\mathrm{CAD}$, the risk reduction after lipid lowering therapy has been found to be not more than $30 \%$ in most of the clinical studies. ${ }^{10}$

These findings led to the search of risk factors in addition to those previously known which could contribute to the development of atherosclerosis and CAD, that is, the "beyond cholesterol" concept. ${ }^{11}$ Evidence started pointing toward the fact that it is in particular the sdLDL which is responsible for its atherogenic property. ${ }^{12}$

\section{LDL and Its Subtypes}

LDL is the main cholesterol carrying lipoprotein. It is broadly defined as lipoprotein fraction with density 1.019-1.063 $\mathrm{g} / \mathrm{mL} .^{13}$ Structurally, it consists of a core of cholesterol esters surrounded by a coat of phospholipids and protein (Apo B100 mainly). LDL delivers cholesterol to the cells, where it is utilized for synthesis of membranes, steroid hormones, etc. Krauss and Blanche used density gradient ultracentrifugation and separated LDL particles of normal subjects according to their size and density. ${ }^{14}$ Austin et al (1990) conducted a study on 301 subjects. They separated LDL using gradient gel electrophoresis and identified two distinct patterns, Pattern A with a predominance of large buoyant LDL particles (density $1.019-1.044 \mathrm{~g} / \mathrm{mL}$ ), and Pattern B consisting of mainly small dense particles (density $1.044-1.063 \mathrm{~g} / \mathrm{mL})^{15}$ (- Fig. 1).

Pattern B (sdLDL) has been found to be associated with number of diseases including CHD, obesity, metabolic syndrome, acute ischemic stroke, and Type 2 diabetes mellitus (DM) ${ }^{16,17}$ High sdLDL itself is a component of "atherogenic phenotype," consisting of high TGs, low HDL, and high sdLDL. This is now recognized as a distinct dyslipidemia linked to various genetic loci. ${ }^{18}$ Accordingly, sdLDL concentration was accepted as a risk factor for cardiovascular events by National Cholesterol Education Program (NCEP III). ${ }^{19}$

\section{Atherogenicity of sdLDL}

The next question which arises is "Why is sdLDL atherogenic?" Small dense LDL differs from large buoyant LDL in certain properties. First, sdLDL has lesser affinity for the LDL receptor than other LDL subtypes. This leads to reduced LDL uptake and as a result, prolonged existence in the circulation. Second, sdLDL has greater affinity for arterial wall proteoglycans. The result is increased retention in the subendothelial space and hence greater opportunity for promoting atherogenic changes. These properties are a result of conformational changes in the Apo B part of sdLDL. These changes alter the properties of sdLDL; the result being decreased receptor-mediated uptake and increased proteoglycan affinity. ${ }^{20}$ Desialylation of sdLDL also contributes to this property. ${ }^{13}$

Oxidized LDL is well known for its atherogenicity. LDL oxidation takes place in the circulation; it leads to the generation of specific epitopes which are recognized by receptors and induce immune response and inflammation. The altered lipid composition and lesser content of antioxidative vitamins reduce the resistance of sdLDL to oxidative stress and hence increase its atherogenic potential. ${ }^{13}$

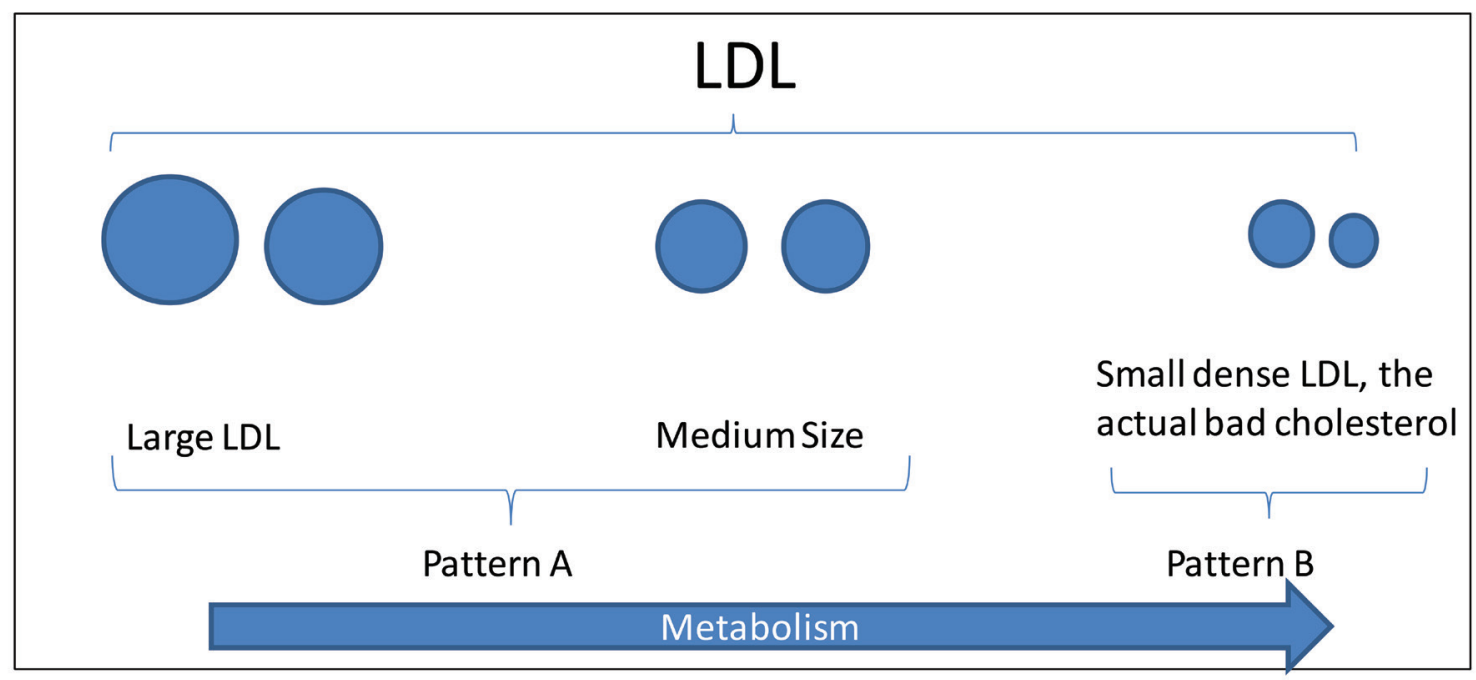

Fig. 1 Low-density lipoprotein and its subtypes. LDL, low-density lipoprotein. 
Numerous studies have proven the atherogenic potential of sdLDL. Nishikura et al in a prospective cohort study of 7 years concluded that sdLDL is a promising biomarker to predict future cardiovascular events in the secondary prevention of stable CAD. ${ }^{6}$ In the Quebec Cardiovascular Study which was a prospective cohort study of 13 years, it was concluded that sdLDL is a strong independent predictor of ischemic heart disease (IHD). ${ }^{21}$ The Framingham Offspring Study which was a prospective cohort study on 1,680 women and 1,508 men concluded that sdLDL is a very strong predictor of IHD in women, whereas in men it is the sdLDL/LDL ratio which is a stronger predictor. ${ }^{17}$

\section{What Determines the Distribution between Large Buoyant and Small Dense LDL?}

SdLDL is a subtype of LDL, so the basic synthetic pathway is the same. Triglyceride (TG)-rich very-low-density lipoprotein (VLDL) is synthesized in the liver and released into the circulation. TGs are acted upon by lipoprotein lipase and hepatic lipase producing intermediate-density lipoprotein and LDL. Simultaneously, LDL is acted upon by cholesterol ester transfer protein (CEPT) which removes cholesterol esters from LDL in exchange for TGs which it brings in from other lipoproteins. The distribution of LDL among large buoyant and small dense subtypes depends on various factors. The trait of sdLDL predominance or the atherogenic lipoprotein phenotype (high TGs, low HDL, and high sdLDL) is a distinct dyslipidemia linked to various genetic loci with a heritability of 35 to $45 \% .{ }^{18}$ The prevalence of this trait is higher in older men and postmenopausal women. Other factors include diet, obesity, exercise, drugs, abdominal adiposity, and hormonal status ${ }^{20}$ ( - Fig. 2).
However, the major factor determining LDL subtype distribution is the level of TGs. Studies have shown that pattern B subclass distribution is not seen till TG levels exceed $1.5 \mathrm{mmol} / \mathrm{L}$ or $120 \mathrm{mg} / \mathrm{dL}$. Fifty percent of the variation in LDL size is determined by TG levels. When TG levels are high, it leads to increased synthesis of TG-rich VLDL1. Lipolysis of VLDL1 produces LDL particles with prolonged residence time of around 5 days in the circulation compared with LDL derived from smaller VLDL precursors which remain in the circulation for around 2 days. This smaller LDL has sufficient time to be remodeled by CETP; LDL loses cholesterol esters and gains TGs. If this happens to a certain degree, next exposure of TG-rich LDL to hepatic lipase (HL) promotes a particle shift to smaller denser range. Hence the levels of activity of CETP and HL also influence LDL subclass distribution ${ }^{22}$ ( - Fig. 3 ).

\section{sdLDL as a Drug Target}

The importance of sdLDL as a predictive biomarker in CAD is well established and its atherogenic potential understood. Numerous studies are now being performed using different approaches of modifying the lipid profile. Instead of LDL, targeting sdLDL alone or along with high TGs and low HDL as a part of the atherogenic triad, can probably give a better outcome. Different approaches have been tested for reducing sdLDL with variable results.

\section{Fibrates}

Since high TGs are a direct influencing factor on sdLDL levels, TG reduction was one of the earliest approaches tried out.

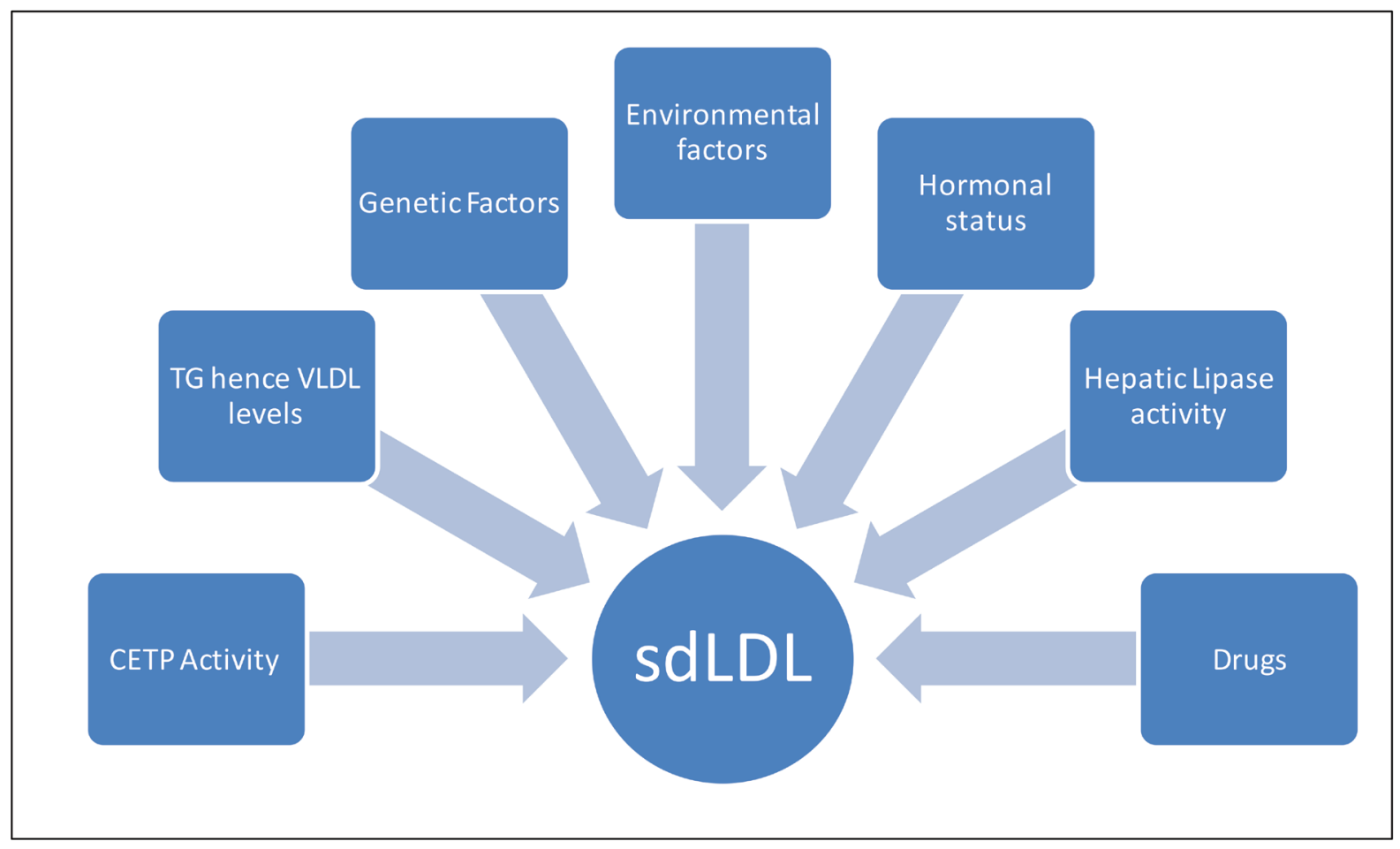

Fig. 2 Factors influencing SdLDL levels. CETP, cholesterol ester transfer protein; SdLDL, small dense low-density lipoprotein; TG, triglycerides; VLDL, very-low-density lipoprotein. 


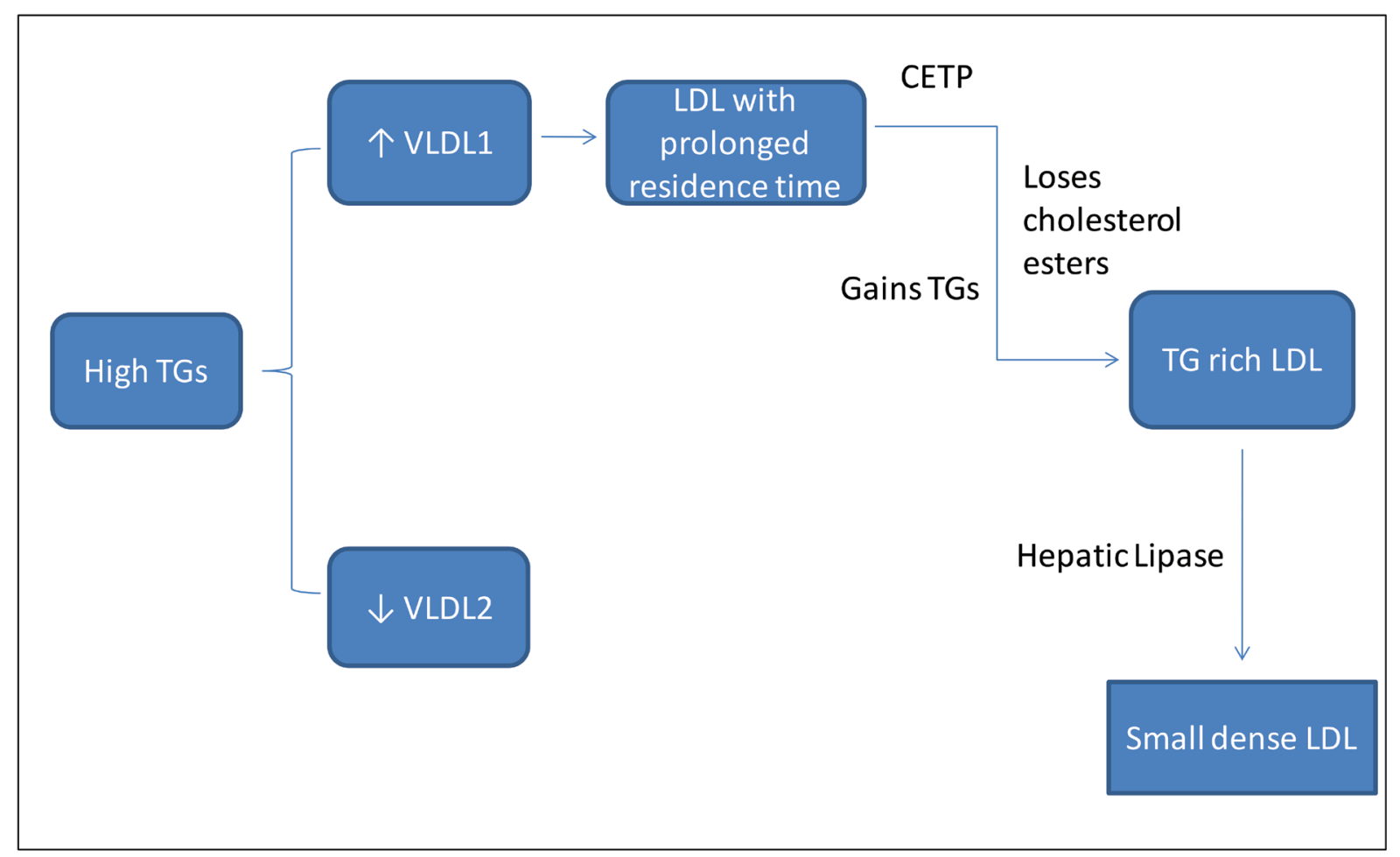

Fig. 3 Pathway of small dense low-density lipoprotein synthesis. CETP, cholesterol ester transfer protein; LDL, low-density lipoprotein; TG, triglycerides; VLDL, very-low-density lipoprotein.

Fibrates are peroxisome proliferator activated receptor $\alpha$ agonists. They increase muscle and hepatic fatty acid oxidation and reduce lipogenesis. In addition, they increase lipoprotein lipase (LPL) activity and reduce VLDL levels through decreased synthesis and increased clearance. The effect is reduced TG levels, modest elevation of HDL, and shift of sdLDL to large buoyant LDL( $(b L D L)$ while total LDL remains the same. Though apparently fibrates appear to be a good treatment modality, disappointing results were obtained in clinical trials in regard to CVD prevention. Drugs that have been tested include fenofibrate, bezafibrate, and gemfibrozil. ${ }^{23-25}$ Meta-analysis of the fibrate outcome trials has revealed that significant positive outcome is obtained in hypertriglyceridemic patients, who are but not in those who are normotriglyceridemic. ${ }^{26} \mathrm{At}$ present, fibrates are being used as an add on to statins in high-risk patients with baseline high TG levels. ${ }^{27}$

\section{Niacin}

Niacin reduces TG levels by decreasing TG synthesis and accelerating Apo B degradation leading to decreased secretion of VLDL, LDL, and sdLDL. High-dose niacin increases HDL. ${ }^{28}$ Immediate release of niacin causes flushing and worsens glycemic control in Type 2 DM patients. Slow release forms are hepatotoxic and less efficient in raising HDL levels. Extended release forms have favorable effect on the lipid profile without being hepatotoxic or worsening glycemic control. ${ }^{27}$ However, no additional benefit of adding niacin to statins has been demonstrated in any clinical trial for improving CVD outcome. ${ }^{29}$

\section{CETP Inhibitors}

CETP mediates transfer of TGs from TG-rich lipoproteins to HDL and LDL in exchange for cholesterol esters. This is the main pathway of reverse cholesterol transport and hence, cholesterol breakdown and excretion through the liver. Use of CETP inhibitors, for example, torcetrapib increases HDL levels but also impairs reverse cholesterol transport, hence curbing its benefit. ${ }^{27}$ Until now good results have not been obtained using CETP inhibitors. Newer more potent drugs are being developed. Anacetrapib is one such drug which increases HDL, reduces LDL and Apo B 100, and increases the LDL TG/ cholesterol ratio, LDL size, and Apo B 100 clearance. $^{30,31}$

\section{Statins}

Statins inhibit the enzyme HMG CoA reductase; hence they control the rate limiting step of cholesterol synthesis. They are highly effective in reducing LDL; they also reduce TGs and raise HDL. Rosuvastatin in high doses is highly effective in reducing sdLDL levels. ${ }^{32}$ Fukushima et al used atorvastatin to reduce LDL in patients with acute coronary syndrome and metabolic syndrome and found that the sdLDL reduction was 5.5 times greater than ACS patients without metabolic syndrome. ${ }^{33}$ Choi et al, however, reported that though statins reduce the absolute levels of both SdLDL and IbLDL, they are not effective in reducing the proportion of sdLDL to total LDL. $^{34}$

\section{Sodium-Glucose Co-transporter-2 Inhibitors}

Sodium-glucose co-transporter-2 (SGLT2) inhibitors are a group of drugs used in the treatment of Type 2 DM. They reduce blood glucose by inhibiting glucose re-absorption in the proximal tubules of the kidney. Clinical trials have revealed that SGLT-2 inhibitors reduce TGs and increase not only HDL but LDL as well. Hayashi et al in a study on the effect of the SGLT2 inhibitor dapagliflozin on the lipid profile of 
Type 2 diabetics reported that dapagliflozin reduced the levels of sdLDL by $20 \%$ and increased IdLDL by $18 \%$ keeping the total LDL levels the same. They postulated that the reduction in sdLDL levels was brought about by reducing TG levels and increasing insulin sensitivity. ${ }^{35}$

\section{Future Prospects}

LDL reduction and subtype redistribution is an area of constant research. Interventional methodologies are being developed which will target the primary pathophysiological aspects of the atherogenic dyslipidemia complex and will lead to HDL increase and LDL subclass modification as a secondary effect. Together this will lead to a better outcome for those patients at risk of CAD. Some of the ongoing research areas are discussed below.

LPL activity is one of the factors influencing sdLDL levels. In patients with homozygous loss of function mutations leading to severe hypertriglyceridemia, gene therapy has a good outcome. ${ }^{36}$ Strategies other than gene therapy are also being explored. APOC3 is an inhibitor of LPL. Inhibition of APOC3 using antisense therapy may reduce sdLDL levels. ${ }^{37}$ ANGPTL are another group of proteins which inhibit LPL. Antibody-mediated blockade of ANGPTL3 has given good outcome in animal models. ${ }^{38}$

Other methods being tried out include inhibition of certain enzymes of lipid metabolism. Monoacylglycerol acyl transferase and diacylglycerol acyl transferase are enzymes involved in TG synthesis. ${ }^{39}$ Acetyl CoA carboxylase catalyzes the rate limiting step in the fatty acid synthesis. Drug-mediated inhibition of these enzymes may decrease lipogenesis and increase lipid oxidation. Clinical trials are going on for these drugs. ${ }^{40}$

ETC-1002 (bempedoic acid) is another drug under investigation. It inhibits adenosine triphosphate (ATP) citrate lyase and activates AMP-activated protein kinase, thus reducing fatty acid synthesis and increasing its oxidation. A major benefit of this drug is that it reduces LDL levels regardless of the TG levels. ${ }^{41,42}$ However, further clinical trials are required to assess its efficacy and safety as a combination drug. ${ }^{27}$

Whatever the treatment strategy being developed in the future, the importance of lifestyle modification in lipid lowering should not be ignored. Diet, exercise, and maintenance of healthy body weight have a positive effect on atherogenic dyslipidemia. Improved-insulin sensitivity and hormonal balance which accompanies lifestyle changes are thought to bring about this effect. ${ }^{43-45}$

\section{Conclusion}

Small dense LDL is the atherogenic component of LDL. It is a predictive biomarker for CAD. Lipid lowering drugs are being routinely used as a treatment component in high-risk patients for primary and secondary prevention of CAD. But the outcome is not always favorable, in spite of significant LDL reduction. Targeting the atherogenic dyslipidemia complex stressing on sdLDL reduction may be the solution. Various drugs have been shown to be effective in reducing sdLDL. However, further research is necessary to draw clear guidelines for sdLDL reduction therapy in CAD treatment and prevention.

\section{Conflict of Interest}

None declared.

\section{References}

1 Mozaffarian D, Benjamin EJ, Go AS, et al. Writing Group Members; American Heart Association Statistics Committee; Stroke Statistics Subcommittee. Executive summary: heart disease and stroke statistics-2016 update: a report from the American Heart Association. Circulation 2016;133(4):447-454

2 Krishnan MN. Coronary heart disease and risk factors in India-on the brink of an epidemic? Indian Heart J 2012;64(4):364-367

3 Gupta R, Mohan I, Narula J. Trends in coronary heart disease epidemiology in India. Ann Glob Health 2016;82(2):307-315

4 Gupta R, Misra A, Vikram NK, et al. Younger age of escalation of cardiovascular risk factors in Asian Indian subjects. BMC Cardiovasc Disord 2009;9:28

5 Prabhakaran D, Jeemon P, Roy A. Cardiovascular diseases in India: current epidemiology and future directions. Circulation 2016;133(16):1605-1620

6 Nishikura T, Koba S, Yokota Y, et al. Elevated small dense low-density lipoprotein cholesterol as a predictor for future cardiovascular events in patients with stable coronary artery disease. J Atheroscler Thromb 2014;21(8):755-767

7 Yusuf S, Hawken S, Ounpuu S, et al. INTERHEART Study Investigators. Effect of potentially modifiable risk factors associated with myocardial infarction in 52 countries (the INTERHEART study): case-control study. Lancet 2004;364(9438):937-952

8 Werner RM, Pearson TA. LDL-cholesterol: a risk factor for coronary artery disease-from epidemiology to clinical trials. Can J Cardiol 1998;14(Suppl B):3B-10B

9 Baigent C, Keech A, Kearney PM, et al. Cholesterol Treatment Trialists' (CTT) Collaborators. Efficacy and safety of cholesterol-lowering treatment: prospective meta-analysis of data from 90,056 participants in 14 randomised trials of statins. Lancet 2005;366(9493):1267-1278

10 Afilalo J, Majdan AA, Eisenberg MJ. Intensive statin therapy in acute coronary syndromes and stable coronary heart disease: a comparative meta-analysis of randomised controlled trials. Heart 2007;93(8):914-921

11 Khalil R, Al-Azab D, Akl OA. Is sdLDL a valuable screening tool for cardiovascular disease in patients with metabolic syndrome? Alex J Med 2017;53(4):299-305

12 Griffin BA, Freeman DJ, Tait GW, et al. Role of plasma triglyceride in the regulation of plasma low density lipoprotein (LDL) subfractions: relative contribution of small, dense LDL to coronary heart disease risk. Atherosclerosis 1994;106(2):241-253

13 Ivanova EA, Myasoedova VA, Melnichenko AA, Grechko AV, Orekhov AN. Small dense low-density lipoprotein as biomarker for atherosclerotic diseases. Oxid Med Cell Longev 2017;2017:1273042

14 Krauss RM, Blanche PJ. Detection and quantitation of LDL subfractions. Curr Opin Lipidol 1992;3(6):377-383

15 Austin MA, Brunzell JD, Fitch WL, Krauss RM. Inheritance of low density lipoprotein subclass patterns in familial combined hyperlipidemia. Arterioscler Dallas Tex 1990;10(4):520-530

16 Zeljkovic A, Vekic J, Spasojevic-Kalimanovska V, et al. LDL and HDL subclasses in acute ischemic stroke: prediction of risk and short-term mortality. Atherosclerosis 2010;210(2):548-554

17 Ai M, Otokozawa S, Asztalos BF, et al. Small dense LDL cholesterol and coronary heart disease: results from the Framingham Offspring Study. Clin Chem 2010;56(6):967-976 
18 Austin MA, King MC, Vranizan KM, Krauss RM. Atherogenic lipoprotein phenotype. A proposed genetic marker for coronary heart disease risk. Circulation 1990;82(2):495-506

19 National Cholesterol Education Program (NCEP) Expert Panel on Detection, Evaluation, and Treatment of High Blood Cholesterol in Adults (Adult Treatment Panel III). Third report of the National Cholesterol Education Program (NCEP) expert panel on detection, evaluation, and treatment of high blood cholesterol in adults (Adult Treatment Panel III) final report. Circulation 2002;106(25):3143-3421

20 Packard CJ. Triacylglycerol-rich lipoproteins and the generation of small, dense low-density lipoprotein. Biochem Soc Trans 2003;31:1066-1069

21 St-Pierre AC, Cantin B, Dagenais GR, et al. Low-density lipoprotein subfractions and the long-term risk of ischemic heart disease in men: 13-year follow-up data from the Québec Cardiovascular Study. Arterioscler Thromb Vasc Biol 2005;25(3):553-559

22 Berneis KK, Krauss RM. Metabolic origins and clinical significance of LDL heterogeneity. J Lipid Res 2002;43(9):1363-1379

23 Keech A, Simes RJ, Barter P, et al. FIELD study investigators. Effects of long-term fenofibrate therapy on cardiovascular events in 9795 people with type 2 diabetes mellitus (the FIELD study): randomised controlled trial. Lancet 2005;366(9500):1849-1861

24 Bezafibrate IPB; Bezafibrate Infarction Prevention (BIP) study. Secondary prevention by raising HDL cholesterol and reducing triglycerides in patients with coronary artery disease. Circulation 2000;102(1):21-27

25 Manninen V, Tenkanen L, Koskinen P, et al. Joint effects of serum triglyceride and LDL cholesterol and HDL cholesterol concentrations on coronary heart disease risk in the Helsinki Heart Study. Implications for treatment. Circulation 1992;85(1):37-45

26 Perkins WJ Jr. Combination lipid therapy in Type 2 diabetes. N Engl J Med 2010;363:692-695

27 Xiao C, Dash S, Morgantini C, Hegele RA, Lewis GF. Pharmacological targeting of the atherogenic dyslipidemia complex: the next frontier in CVD prevention beyond lowering LDL cholesterol. Diabetes 2016;65(7):1767-1778

28 Bruckert E, Labreuche J, Amarenco P. Meta-analysis of the effect of nicotinic acid alone or in combination on cardiovascular events and atherosclerosis. Atherosclerosis 2010;210(2):353-361

29 Boden WE, Probstfield JL, Anderson T, et al. AIM-HIGH Investigators. Niacin in patients with low HDL cholesterol levels receiving intensive statin therapy. $N$ Engl J Med 2011;365(24):2255-2267

30 Bloomfield D, Carlson GL, Sapre A, et al. Efficacy and safety of the cholesteryl ester transfer protein inhibitor anacetrapib as monotherapy and coadministered with atorvastatin in dyslipidemic patients. Am Heart J 2009;157(2):352-360

31 Teramoto T, Numaguchi H, Shirakawa M, et al. Efficacy and safety of the cholesteryl ester transfer protein inhibitor anacetrapib as monotherapy and coadministered with atorvastatin in Japanese patients with dyslipidemia. J Am Coll Cardiol 2012;59(13, Suppl):E1689
32 Nishikido T, Oyama J, Keida T, Ohira H, Node K. High-dose statin therapy with rosuvastatin reduces small dense LDL and MDALDL: the Standard versus high-dose therApy with Rosuvastatin for lipiD lowering (SARD) trial. J Cardiol 2016;67(4):340-346

33 Fukushima Y, Hirayama S, Ueno T, et al. Small dense LDL cholesterol is a robust therapeutic marker of statin treatment in patients with acute coronary syndrome and metabolic syndrome. Clin Chim Acta 2011;412(15-16):1423-1427

34 Choi CU, Seo HS, Lee EM, et al. Statins do not decrease small, dense low-density lipoprotein. Tex Heart Inst J 2010;37(4): 421-428

35 Hayashi T, Fukui T, Nakanishi N, et al. Dapagliflozin decreases small dense low-density lipoprotein-cholesterol and increases high-density lipoprotein 2-cholesterol in patients with type 2 diabetes: comparison with sitagliptin. Cardiovasc Diabetol 2017; 16(1):8

36 Gaudet D, Méthot J, Déry S, et al. Efficacy and long-term safety of alipogene tiparvovec (AAV1-LPLS447X) gene therapy for lipoprotein lipase deficiency: an open-label trial. Gene Ther 2013;20(4):361-369

37 Graham MJ, Lee RG, Bell TA, III. et al. Antisense oligonucleotide inhibition of apolipoprotein C-III reduces plasma triglycerides in rodents, nonhuman primates, and humans. Circ Res 2013;112(11):1479-1490

38 Wang Y, Gusarova V, Banfi S, Gromada J, Cohen JC, Hobbs HH. Inactivation of ANGPTL3 reduces hepatic VLDL-triglyceride secretion. J Lipid Res 2015;56(7):1296-1307

39 Shi Y, Cheng D. Beyond triglyceride synthesis: the dynamic functional roles of MGAT and DGAT enzymes in energy metabolism. Am J Physiol Endocrinol Metab 2009;297(1):E10-E18

40 Griffith DA, Kung DW, Esler WP, et al. Decreasing the rate of metabolic ketone reduction in the discovery of a clinical acetyl-CoA carboxylase inhibitor for the treatment of diabetes. J Med Chem 2014;57(24):10512-10526

41 Pinkosky SL, Filippov S, Srivastava RAK, et al. AMP-activated protein kinase and ATP-citrate lyase are two distinct molecular targets for ETC-1002, a novel small molecule regulator of lipid and carbohydrate metabolism. J Lipid Res 2013;54(1):134-151

42 Ballantyne CM, Davidson MH, Macdougall DE, et al. Efficacy and safety of a novel dual modulator of adenosine triphosphate-citrate lyase and adenosine monophosphate-activated protein kinase in patients with hypercholesterolemia: results of a multicenter, randomized, double-blind, placebo-controlled, parallel-group trial. J Am Coll Cardiol 2013;62(13):1154-1162

43 Purnell JQ Kahn SE, Albers JJ, Nevin DN, Brunzell JD, Schwartz RS. Effect of weight loss with reduction of intra-abdominal fat on lipid metabolism in older men. J Clin Endocrinol Metab 2000;85(3):977-982

44 Siri-Tarino PW, Woods AC, Bray GA, Krauss RM. Reversal of small, dense LDL subclass phenotype by weight loss is associated with impaired fat oxidation. Obesity (Silver Spring) 2011;19(1):61-68

45 Siri-Tarino PW, Williams PT, Fernstrom HS, Rawlings RS, Krauss RM. Reversal of small, dense LDL subclass phenotype by normalization of adiposity. Obesity (Silver Spring) 2009;17(9):1768-1775 